\title{
Cardiovascular Disease Risk among Rural Population of South India
}

\section{BADAL CHANDRA BHAKAT*1, K. VANI MADHAVI ${ }^{2}$}

INTRODUCTION: The leading causes of death and disability both in the developed and developing nations is Cardiovascular Disease with its major manifestations are Coronary Artery Disease (CAD) and stroke.

AIM: To assess the cardiovascular disease risk in adults aged 40-6oyears and find out the significant predictors associated with cardiovascular risk among the rural adult population.

MATERIALS AND METHOD: The study was a cross-sectional community based study in the rural field practice area of KIMS, Amalapuram and included a sample of 150 subjects ( 75 males and 75 females) of age 40-6o years. A Pre-tested, semi structured questionnaire was used to obtain relevant information from participants about their demographics and health habits. The Framingham Risk Score that consists of age, sex, total cholesterol, HDL-cholesterol, blood pressure, and smoking, to calculate the 10-year risk probability of developing the cardiovascular disease was used to collect the data. Physical examination and blood samples were taken from those participants and Epi-info statistical software package version 3.5.4 was used for data analysis.

RESULTS: According to Framingham risk score in our study most of the risk of cardiovascular diseases was more for males. Among low risk group males were $43 \%$, females $86 \%$ and in medium risk group males were $17 \%$, females $8 \%$ where as in high risk group majority were males (40\%) compared to females $(6 \%)$ and it was found to be statistically significant.

CONCLUSION: The Framingham Risk Score can help identify the risk group for Cardiovascular Disease Risk, and shall help educate the individual as well as community for lifestyle modification.

KEYWORDS: Framingham Risk Score, Coronary Artery Disease (CAD), Cardiovascular Disease, Stroke

\section{INTRODUCTION}

Cardiovascular diseases are the leading causes of death and disability both in the developed and developing nations. The two major manifestations are, CAD and stroke. Global estimate of mortality due to cardiovascular disease for the year 2008, was 17 million, $48 \%$ of all NCD deaths. ${ }^{1}$

Within the turn of the century cardiovascular disease have become the leading cause of mortality in India. In comparison with people of European ancestory, CVD affects Indian, at least a decade earlier and in their most productive midlife years. ${ }^{2}$ The incidence of CVD is greater in urban areas than in rural areas, reflecting the acquisition of several risk factors such as tobacco consumption, lack of physical activity, unhealthy diet, and obesity.

Early detection and prediction of risk of developing the cardiovascular disease is a crucial factor in slowing the growth of cardiovascular-related mortality across the world. ${ }^{3}$ The Framingham heart study resulted in the development of the Framingham risk score for coronary heart disease (CHD), which used age, sex, total cholesterol, high- density lipoprotein (HDL) cholesterol, blood pressure (and also whether the patient is treated or not for his/her hypertension), and smoking to calculate the 10-year risk probability of developing cardiovascular disease for a given patient. ${ }^{4}$

There is, an urgent need for focused health programs that are targeted to both reduce risk and increase early risk detection of cardiovascular disease in this population. The aim of this study is to assess the 10 years cardiovascular risk according to the Framingham risk score in the rural area of South India.

\section{OBJECTIVES}

1. To assess the cardiovascular disease risk in adults aged 40-6o years among the rural population.

2. To find out the significant predictors associated with cardiovascular risk among the rural adult population.

\section{MATERIALS AND METHODS}

Study Design: Cross sectional community based study. 
Study Setting: Rural field practice area of KIMS, Amalapuram.

Sample Size: 150 subjects ( 75 males and 75 females) of age $40-60$ years.

(I) Inclusion Criteria: Adults in the age group of 406oyears, who are residing in the selected area, available at the time of visit, willing to participate and consented will be included in the study

\section{(II) Exclusion Criteria:}

(a) Pregnant women.

(b) significant physical disability.

(c) Pre-existing cardiovascular disease, cerebrovascular disease, chronic kidney disease,diabetes \& mental disorder patients.

(III) Time Period: July 2016 - November 2016

\section{DATA COLLECTION PROCEDURE}

A Pre-tested, semi structured questionnaire was used to obtain relevant information from participants about their demographics and health habits.

We have used the Framingham Risk Score that consists of age, sex, total cholesterol, HDLcholesterol, blood pressure, and smoking, to calculate the 10-year risk probability of developing the cardiovascular disease.
Physical examination was conducted for height, weight, abdominal circumference, systolic and diastolic blood pressure using standard WHO guidelines.

Blood samples were taken from those participants, for biochemical investigation. Total cholesterol, HDLcholesterol, LDL cholesterol and, blood glucose were measured according to standard clinical laboratory procedure. $^{5}$

Study Variables: Age, educational status, marital status, income, consumption of fruits, red meat, junk food, salt intake, Physical activity, BMI, abdominal circumference, blood pressure, HDL and total cholesterol.

Statistical Analysis: Epi-info statistical software package version 3.5.4 was used for data analysis and $P$ value $<0.05$ was considered statistically significant.

Ethical consideration: Institutional ethics committee (IEC) approval was taken, informed written consent was obtained from the participants.

\section{RESULTS}

Of the 150 participants (average age 49.03 \pm 7.1 ), detail characteristics of behaviour, anthropometric, and biochemical risk factor are summarized in Table 1.

\begin{tabular}{|c|c|c|c|}
\hline $\begin{array}{c}\text { STUDY VARIABLES } \\
\text { Hypertension }\end{array}$ & MALE & FEMALE & p-value \\
\hline Obesity & $31(41.3 \%)$ & 0.01 \\
\hline High Total cholesterol & $22(29.3 \%)$ & $26(34.6 \%)$ & 0.18 \\
\hline Low-HDL- Cholesterol & $10(13.5 \%)$ & $12(15.8 \%)$ & 0.86 \\
\hline Sedentary Habits & $13(17.3 \%)$ & $74(98.6 \%)$ & 0.00 \\
\hline Smoking & $13(17.6 \%)$ & $3(3.95 \%)$ & 0.01 \\
\hline
\end{tabular}

Table 1. Characteristics of behaviour, anthropometric, and biochemical risk factor of the study population. $* p$ value $<0.05$ significant

According to Framingham risk score in our study most of the risk of cardiovascular diseases was more for males. Among low risk group males were 43\%, females $86 \%$ and in medium risk group males were 


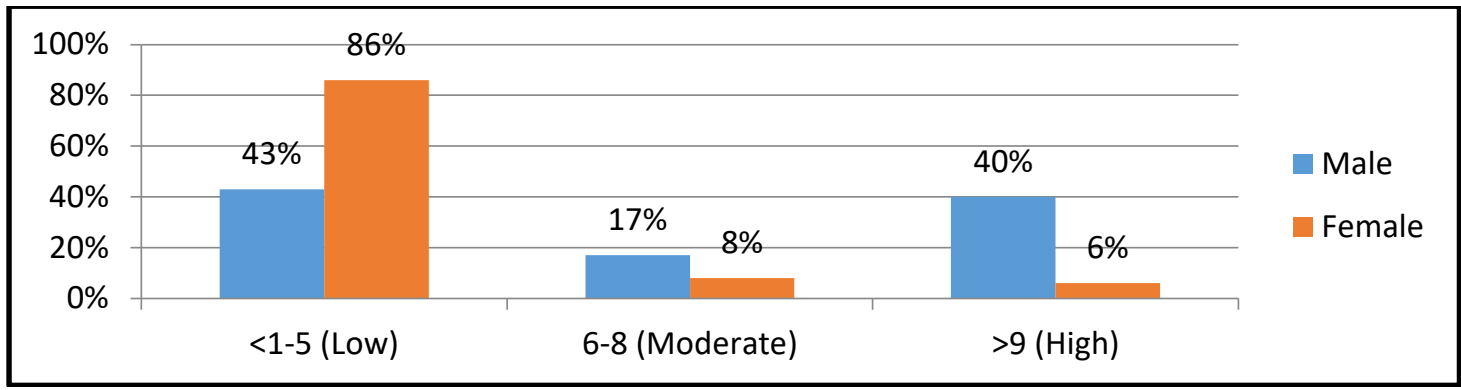

Figure 1. Framingham Risk Score Distribution

$17 \%$, females $8 \%$ where as in high risk group majority were males $(40 \%)$ compared to females $(6 \%)$ and it was found to be statistically significant.

\section{DISCUSSION}

The study conducted at Kochi (Amrita NIRAM study $)^{6}$ in the population attending the Amrita comprehensive health clinic, urban area with age group of 40-59 years using the Framingham risk score identified $30 \%$ with moderate risk and $12 \%$ with high risk of future cardiovascular end points.

Whereas in contrast to that, our present study showed $64.67 \%$ of low risk group and $12.67 \%$ of moderate risk group and $22.66 \%$ of high risk, as our study was conducted in rural area. We have observed participants with $(48 \%)$ pre hypertension and $10.6 \%$ with low physical exercises highlighting the need for intervention.

\section{CONCLUSION}

By using the Framingham Risk Score we can identify the risk group, it will help us to educate the individual as well as community for lifestyle modification.

\section{REFERENCES}

1. WHO: Global Status Report on Non Communicable diseases. 2010

2. Xavier D, Pais P, Devereaux PJ, Xie C, Prabhakaran D, Reddy KS, Gupta R, Joshi P, Kerkar P, Thanikachalam S, Haridas KK, Jaison TM, Naik S, Maity AK, Yusuf S; CREATE registry investigators. Treatment and outcomes of acute coronary syndromes in India (CREATE): a prospective analysis of registry data. Lancet. 2008;371:1435-42.

3. Goff DC jr, Loyd-jones DM,BennetG,Coady S,D’AgostinoRBSr, Gibbons R et.al., 2013ACC/AHA guidelines on the assessment of cardiovascular risk, A report of the ACC/AHA Task force on practice guidelines, Journel Am Coll cardiol 2014;463 (25Pt B):2935-59.

4. Kannel,WB Mc Gee D,Gordon T.Ageneral cardiovascular risk profile: The Framingham study Am J Cardiol 1976; 38:46-51.

5. Allain CC, Poon LS, Chan CS, Richmond W, Fu PC. Enzymatic determination of total serum cholesterol. Clin Chem 1974;20:470-5.

6. Menon, Vidya P, et.al., Assessment of 2013 AHA/ACC ASCVD risk scores with behavioral characteristics of an urban cohort in India: Preliminary analysis of Noncommunicable disease initiatives and Research at Amrita (NIRAM) study. Lipincott William Wilkins J. 2016;95:e5542.

\section{AUTHOR AFFILIATIONS:}

1. Final Year PG, KIMS, Amalapuram, East Godavari District, Andhra Pradesh (Corresponding Author)

2. Professor \& HOD, Department of Community Medicine, KIMS, Amalapuram, East Godavari District, Andhra Pradesh.

For article enquiry/author contact details, e-mail at: editor.ihrj@gmail.com,editor@ihrjournal.com 